\title{
Increased Serum Levels of Oxidative Stress Markers in Patients with Liver Steatosis
}

Maria Notarnicola1 ${ }^{1}$, Valeria Tutino ${ }^{1}$, Alberto R Osella², Caterina Bonfiglio², Vito Guerra² and Maria Gabriella Caruso ${ }^{1 *}$

${ }^{1}$ Laboratory of Nutritional Biochemistry, Castellana Grotte (BA), Italy

'Laboratory of Epidemiology and Biostatistic, National Institute for Digestive Diseases "Saverio de Bellis", Castellana Grotte (BA), Italy

\begin{abstract}
Background: Several studies have suggested that oxidative stress could play a role in the pathogenesis of nonalcoholic fatty liver disease and more precisely in the transition between simple fatty liver and steatohepatitis.

Aim: This study aims to investigate if circulating levels of oxidative stress markers could be clinically associated with liver steatosis.

Materials and methods: We present data obtained from a subsample of 70 subjects with liver steatosis enrolled by a nutritional trial, called NUTRIEPA study. Serum levels of oxidative stress markers were evaluated by ELISA assay. The diagnosis and the degree of liver steatosis were based on laboratory and ecographic measurements. Statistical methods included Kruskal-Wallis analysis of variance and, Wilcoxon signed-rank or Mann-Whitney test, where appropriate. The $\mathrm{x} 2$ test has been performed to analyze categorical variables.
\end{abstract}

Results: The subjects with severe or moderate steatosis had significantly higher serum levels of oxidative stress markers compared to subjects without steatosis.

Conclusions: Increased serum levels of oxidative stress markers could be considered a marker of moderate and severe liver steatosis.

\section{Keywords: Oxidative stress markers; Hepatic steatosis}

\section{Introduction}

Liver steatosis is characterized by an excess accumulation of triglycerides within hepatocytes. Excess hepatic fat accumulation could result from the following mechanisms: a) increased de novo fatty acid synthesis; b) decreased fatty acid oxidation; c) increased transport of fatty acids from the peripheral organs to the liver; d) blunted transport of fatty acids (triglycerides) from the liver to the general circulation and peripheral organs [1]. Steatosis is reversible and it has been considered as a benign condition for a long time; however, increasing evidence suggests that it is a potentially pathological condition. If the causal agent is not eliminated or injury persists, steatosis may progress to inflammation, fibrosis and even cirrhosis of the liver, especially in the presence of hepatitis $C$ virus, diabetes and obesity [2-4].

Considering its potential clinical relevance, researchers were prompted to discern the underlying mechanisms of steatosis. Liver steatosis is frequent in patients with metabolic syndrome and accumulating evidences suggest that lipid metabolism is as important to diabetes as carbohydrate metabolism [5]. Recently, high serum levels of Fatty Acid Synthase (FAS) have been detected in patients with chronic hepatitis viral infections and circulating FAS concentration correlated with the degree of liver steatosis $[6,7]$.

Some studies have suggested that, apart from endotoxins, cytokines, hyperlipidemia and hyperinsulinemia, oxidative stress could play a role in the pathogenesis of Nonalcoholic Fatty Liver Disease (NAFLD) and more precisely in the transition between simple fatty liver and steatohepatitis (i.e. fatty liver coexistent with hepatocyte necrosis and inflammation) [8]. Indeed, excessive fat accumulation in the liver, whatever its cause, is prone to attack by Reactive Oxygen Species (ROS), leading to lipid peroxidation with its cellular consequences. For example, increased lipid peroxidation has been documented in animal models, either drug-induced $[9,10]$ or dietary $[11,12]$ while in genetic models of steatosis, such as the ob/ob mice there is an increase in mitochondrial ROS production [13]. Few studies have documented the relationship between the extent of steatosis and systemic markers of oxidative stress. Two recent studies demonstrated different results in circulating levels of oxidative stress markers in NAFLD patients compared with age-matched controls $[14,15]$. Therefore in the present study we have determined whether blood oxidative stress parameters are related to the presence of liver steatosis and could serve as markers of liver fat accumulation at different grade.

\section{Methods}

\section{Patients}

This work is part of NUTRIEPA study, a nutritional trial enrolling consecutive subjects with liver steatosis, diagnosed by abdominal ultrasound.

In this report, we present data obtained from a subsample of 70 subjects. All subjects underwent a complete medical examination including weight and height measurements. Body Mass Index (BMI) was calculated as weight in kilograms divided by the square of the height in meters $\left(\mathrm{kg} / \mathrm{m}^{2}\right)$. Participants were fasted for $12 \mathrm{~h}$ prior to examination. Blood samples taken from the subjects by venous

*Corresponding author: Maria Gabriella Caruso, MD, Laboratory of Nutritional Biochemistry, National Institute for Digestive Diseases "Saverio de Bellis", Via Turi 27, 70013 Castellana Grotte (BA), Italy, Tel: +39 0804994669; Fax: +39 0804994313; E-mail: gabriella.caruso@irccsdebellis.it

Received August 26, 2013; Accepted September 05, 2013; Published September 13,2013

Citation: Notarnicola M, Tutino V, Osella AR, Bonfiglio C, Guerra V, et al. (2013) Increased Serum Levels of Oxidative Stress Markers in Patients with Liver Steatosis. J Liver 2: 127. doi:10.4172/2167-0889.1000127

Copyright: (c) 2013 Notarnicola M, et al. This is an open-access article distributed under the terms of the Creative Commons Attribution License, which permits unrestricted use, distribution, and reproduction in any medium, provided the original author and source are credited. 
puncture were collected in tubes containing a serum separator gel. Aliquots of blood serum were stored at $-80^{\circ} \mathrm{C}$ until assayed or shipped to the central laboratory for routine analyses. All the analyses were performed within 6 months.

In order to obtain a semi quantitative evaluation of fat in the liver, we used a system scoring [16,17]. Degree of fatty infiltration of the liver was graded according to ultrasonographic appearance of liver echotexture, hepatic echo penetration and clarity of hepatic blood vessels, liver diaphragm differentiation in echo amplitude. Each criterion was assigned a score indicating the level of fatty liver infiltration. A score of 2 indicated a definite positive $(++)$ fatty liver infiltration for that criterion. A score of 1 was assigned for a probable positive (+) finding based on the criterion. When a negative (-) evaluation for fatty liver was made from a criterion, a score of 0 was assigned to the criterion. The sum of the scores for the three criteria was considered to be an indicator of severity of fatty infiltration. Thus, the fatty liver indicator ranged from 0 to 6 , with mild fat infiltration for a total score 1-2; moderate for a total score of 2-4, severe indicated by a total score $\geq 5$.

Moreover, a control group of 19 subjects without liver steatosis was included. In this group, the liver steatosis was excluded by ultrasonographic and laboratory evaluation.

The study had institutional approval and all patients gave informed consent to participate in the study.

\section{Laboratory measurements}

In order to estimate the HOMA index, serum levels of glucose and insulin were measured by an automated system (Uni Cel Integrated Workstations DxC 660i, Beckman Coulter, Fullerton, California, USA) using chemioluminescent immunometric method. Samples over calibration range were automatically reanalyzed according to constructor's instructions.

Serum levels of CuZn-Superoxide-Dismutase (CuZnSOD, Abnova, Taipei, Taiwan) and LDL-oxidized (Mercodia, Uppsala, Sweden) were determined by ELISA kits, while serum levels of Thiobarbituric Acid Reactive Substances (TBARS, Cell Biolabs, Inc, San Diego, CA)) were spectrophotometrically analyzed by the measure of MDA quantitation.

\section{Statistical analysis}

The significance of the differences among the groups was evaluated by Kruskal-Wallis analysis of variance, Wilcoxon signed-rank or Mann-Whitney test, where appropriate, and $\chi^{2}$ test for trend. The $\chi^{2}$ test has been performed to analyze categorical variables. All data were expressed as mean $\pm \mathrm{SD}$. Differences were considered significant at $\mathrm{P}$ $<0.05$.

\section{Results}

Liver steatosis was diagnosed and its grade was assessed with the findings of liver ultrasound scan performed by the same radiologist. We found 48 subjects with moderate liver steatosis and 22 with severe steatosis.

Table 1 shows descriptive analysis of studied variables in controls and subjects stratified for the degree of liver steatosis. No difference among the groups was detected in relation to sex and age. The value of BMI and HOMA among the groups was significantly different and the test for trend was also significant $(\mathrm{P}<0.001$ for both parameters). The subjects with steatosis showed circulating levels of LDL-oxidized and TBARS significantly higher than controls (Wilcoxon rank sum-test,
$\mathrm{P}=0.0001$ for both). There are no differences in CuZnSOD serum levels between subjects with steatosis and healthy subjects.

Moreover, stratifying for score of steatosis, the subjects with moderate or severe steatosis had significantly higher serum levels of TBARS and LDL-oxidized compared to subjects without steatosis (Wilcoxon rank sum-test, $\mathrm{P}<0.05$ ). In addition, a positive and statistically significant trend in serum LDL-oxidized concentration from lower to higher degree of steatosis was also detected $(\mathrm{P}<0.001)$.

\section{Discussion}

In this study we describe a relationship between human liver steatosis and elevated levels of circulating stress oxidative markers such as LDL-oxidized and TBARS. The principal findings of the present study are a rise in systemic levels of TBARS and LDL-oxidized in patients with moderate or severe steatosis as compared to healthy controls, while the levels of antioxidant CuZn-SOD protein are similar in both groups, suggesting that serum TBARS and LDL-oxidized measurements may add substantial information for the evaluation of liver impairment.

The over-expression of LDL-oxidized is associated with the degree of liver steatosis because of a positive trend was detected in serum levels of LDL-Oxidized from lower to higher degree of steatosis.

Although liver biopsies and nuclear magnetic resonance imaging are the methods of choice for diagnosis of liver steatosis, ultrasonography is currently the preferred method for screening asymptomatic patients with suspected liver injury [18]. Several studies have demonstrated that the sensitivity, specificity and positive predictive value of this technique to detect steatosis as high as $80-100 \%$ [19-21]. Taking into account for the ethical concerns, we could not perform liver biopsy of our patients. However, in the light of clinical and laboratory parameters, the diagnosis of steatosis was clearly demonstrated.

Some studies showed that products of lipid peroxidation are increased [22-25] and levels of endogenous antioxidants are decreased in patients with NAFLD [26]. Oxidative stress is due to a discordance in balance between prooxidant and antioxidant factors. Normally, a rise in oxidative stress concomitantly enhances the antioxidative activity to protect the cell from damage. Chronic exposure to increased levels of oxidative stress may results in an excess of Reactive Oxygen Species (ROS) within the hepatocytes and starting a vicious cycle [27]. Although a previous study reported that the activity of antioxidant enzymes is increased in NAFLD patients [14], we demonstrated that the antioxidant status in patients with steatosis is apparently incapable to compensate for oxidative stress. It seems highly probable that steatosis-induced lipid peroxidation and ROS consume antioxidant enzymes. Consequently, there is an uncontrolled increase in free oxygen radicals, or an insufficiency in the antioxidant system under certain pathological states. Liver diseases associated with hepatic fat accumulation could amplify the production of ROS because liver fat may serve as a substrate for lipid peroxidation. In a previous study, we have found an increased serum level of lipogenic enzymes, such as FAS and Lipoprotein Lipase (LPL) in patients with liver steatosis [7]. FAS and LPL are central enzymes of lipid metabolism. FAS is believed to be a major determinant of the maximal hepatic capacity to generate fatty acids by de novo lipogenesis [28] and LPL generates free fatty acids that induce insulin resistance in muscle and liver [29]. It has been suggested that insulin resistance is an essential requirement for the accumulation of hepatocellular fat [30].

There are evidences in literature that disturbances in BMI, body 


\begin{tabular}{|c|c|c|c|c|c|c|}
\hline & \multirow[b]{2}{*}{ Controls } & \multicolumn{2}{|c|}{ Steatosis } & & \multirow[b]{2}{*}{ Contrasts } & \multirow[b]{2}{*}{ Test for trend } \\
\hline & & Moderate & Severe & & & \\
\hline & $(n=19)$ & $(n=48)$ & $(n=22)$ & $\mathrm{p}^{\# 0}$ & $p^{\S}$ & $p$ \\
\hline Sex (\%) & & & & 0.20 & & \\
\hline Female & $8(42.1)$ & $12(25.0)$ & $4(18.2)$ & & & \\
\hline Males & $11(57.9)$ & $36(75.0)$ & $18(81.8)$ & & & \\
\hline Age (year) $(M \pm S D)$ & $58.2 \pm 9.8$ & $58.6 \pm 11.4$ & $58.9 \pm 11.0$ & 0.4 & & \\
\hline \multirow[t]{3}{*}{ BMI $\left(\mathbf{k g} / \mathrm{m}^{2}\right)$} & $25.7 \pm 3.2^{\mathrm{ab}}$ & $31.5 \pm 4.3^{\mathrm{ac}}$ & $34.0 \pm 4.9^{\mathrm{bc}}$ & 0.0001 & $\mathrm{a}<0.0001$ & $<0.001$ \\
\hline & & & & & ${ }^{b}<0.0001$ & \\
\hline & & & & & ${ }^{\circ} 0.01$ & \\
\hline \multirow[t]{3}{*}{ HOMA Index } & $1.7 \pm 1.1^{\mathrm{ab}}$ & $5.3 \pm 11.4^{\mathrm{ac}}$ & $4.0 \pm 1.9^{b c}$ & 0.0001 & a $<0.0001$ & $<0.001$ \\
\hline & & & & & $\mathrm{b}<0.0001$ & \\
\hline & & & & & ${ }^{\circ} 0.64$ & \\
\hline \multirow[t]{3}{*}{ LDL OX (U/L) } & $7.6 \pm 1.1^{\mathrm{ab}}$ & $9.0 \pm 1.3^{\mathrm{ac}}$ & $10.2 \pm 1.2^{\mathrm{bc}}$ & 0.0001 & $\mathrm{a}<0.0001$ & 0.001 \\
\hline & & & & & b 0.0009 & \\
\hline & & & & & ${ }^{\circ} 0.77$ & \\
\hline Cu/Zn SOD (ng/ml) & $106.7 \pm 22.8$ & $116.9 \pm 36.4$ & $115.8 \pm 27.4$ & 0.79 & & 0.59 \\
\hline \multirow[t]{3}{*}{ TBARS $(\mu \mathrm{M})$} & $43.9 \pm 23.6^{a b}$ & $158.2 \pm 90.3^{\mathrm{ac}}$ & $153.7 \pm 109.5^{\mathrm{bc}}$ & 0.0001 & $\mathrm{a}<0.0001$ & 0.001 \\
\hline & & & & & b 0.0001 & \\
\hline & & & & & ${ }^{\circ} 0.63$ & \\
\hline
\end{tabular}

\#Chi-square test for categorical variable; ${ }^{\circ}$ Kruskal-Wallis test for continuous variable; $§$ Wilcoxon rank-sum (Mann-Whitney) test

Table 1: Descriptive analysis of the studied variables in controls and in patients with liver steatosis stratified for score. Data are expressed as mean and standard deviation or (percentage).

fat, and lipid metabolism may contribute to altered oxidative status in NAFLD and insulin resistance may be related to decreased antioxidant activity as well as an increase of lipid peroxidation [31,32].

In this view, our findings support these evidences. In fact high levels of LDL-oxidized and TBARS detected in subjects with steatosis are in agreement with the presence of insulin resistance evaluated in same subjects. Moreover, the patients with severe liver steatosis showed a value of BMI significantly higher, demonstrating that some individuals develop metabolic complications in relation to their degree of obesity. Already, in clinical practice high concentrations of circulating LDLoxidized are observed in patients with NAFLD [33].

However, although our results suggest interesting correlations, the different relationships must be taken with caution. As parameters of oxidative stress in serum do not reflect intrahepatic peroxidation, the exact relation between hepatic and serum levels of oxidative stress parameters needs further elucidation.

The liver steatosis may progress to cirrhosis and hepatocellular carcinoma [32]. Therefore, it is clinically important that specific biomarkers of oxidative stress are identified in patients with hepatic steatosis in order to assess the efficacy of diagnosis and therapeutic treatment

\section{References}

1. Anty R, Lemoine M (2011) Liver fibrogenesis and metabolic factors. Clin Res Hepatol Gastroenterol 35: S10-20.

2. Adams LA, Feldstein AE (2011) Non-invasive diagnosis of nonalcoholic fatty liver and nonalcoholic steatohepatitis. J Dig Dis 12: 10-16.

3. Marchesini G, Bugianesi E, Forlani G, Cerrelli F, Lenzi M, et al. (2003) Nonalcoholic fatty liver, steatohepatitis, and the metabolic syndrome. Hepatology 37: 917-923.

4. Erickson SK (2009) Nonalcoholic fatty liver disease. J Lipid Res 50: S412-416.

5. Fernandez-Real JM, Menendez JA, Moreno-Navarrete JM, Blüher M, VazquezMartin A, et al. (2010) Extracellular fatty acid synthase: a possible surrogate biomarker of insulin resistance. Diabetes 59: 1506-1511.
6. Joven J, Espinel E, Rull A, Beltrán-Debón R, Aragonès G, et al. (2011) Serum fatty acid synthase concentration is increased in patients with hepatitis vira infection and may assist in the prediction of liver steatosis. J Clin Virol 51: 199-201.

7. Notarnicola M, Misciagna G, Tutino V, Chiloiro M, Osella AR, et al. (2012) Increased serum levels of lipogenic enzymes in patients with severe liver steatosis. Lipids Health Dis 11: 145

8. Day CP, James OF (1998) Hepatic steatosis: innocent bystander or guilty party? Hepatology 27: 1463-1466.

9. Letteron P, Fromenty B, Terris B, Degott C, Pessayre D (1996) Acute and chronic hepatic steatosis lead to in vivo lipid peroxidation in mice. $J$ Hepatol 24: $200-208$

10. Berson A, De Beco V, Lettéron P, Robin MA, Moreau C, et al. (1998) Steatohepatitis-inducing drugs cause mitochondrial dysfunction and lipid peroxidation in rat hepatocytes. Gastroenterology 114: 764-774.

11. Grattagliano I, Vendemiale G, Caraceni P, Domenicali M, Nardo B, et al. (2000) Starvation impairs antioxidant defense in fatty livers of rats fed a cholinedeficient diet. J Nutr 130: 2131-2136.

12. Oliveira CP, da Costa Gayotto LC, Tatai C, Della Bina BI, Janiszewski M, et al. (2002) Oxidative stress in the pathogenesis of nonalcoholic fatty liver disease, in rats fed with a choline-deficient diet. J Cell Mol Med 6: 399-406.

13. Yang SQ, Lin HZ, Lane MD, Clemens M, Diehl AM (1997) Obesity increases sensitivity to endotoxin liver injury: implications for the pathogenesis of steatohepatitis. Proc Natl Acad Sci U S A 94: 2557-2562.

14. Yesilova Z, Yaman H, Oktenli C, Ozcan A, Uygun A, et al. (2005) Systemic markers of lipid peroxidation and antioxidants in patients with nonalcoholic Fatty liver disease. Am J Gastroenterol 100: 850-855.

15. Bonnefont-Rousselot D, Ratziu V, Giral P, Charlotte F, Beucler I, et al. (2006) Blood oxidative stress markers are unreliable markers of hepatic steatosis. Aliment Pharmacol Ther 23: 91-98.

16. Tominaga K, Kurata JH, Chen YK, Fujimoto E, Miyagawa S, et al. (1995) Prevalence of fatty liver in Japanese children and relationship to obesity. An epidemiological ultrasonographic survey. Dig Dis Sci 40: 2002-2009.

17. Chiloiro M, Riezzo G, Chiarappa S, Correale M, Guerra V, et al. (2008) Relationship among fatty liver, adipose tissue distribution and metabolic profile in moderately obese children: an ultrasonographic study. Curr Pharm Des 14 2693-2698. 
Citation: Notarnicola M, Tutino V, Osella AR, Bonfiglio C, Guerra V, et al. (2013) Increased Serum Levels of Oxidative Stress Markers in Patients with Liver Steatosis. J Liver 2: 127. doi:10.4172/2167-0889.1000127

Page 4 of 4

18. Babali A, Cakal E, Purnak T, Biyikoglu I, Cakal B, et al. (2009) Serum Îtfetoprotein levels in liver steatosis. Hepatol Int 3: 551-555.

19. Hamaguchi M, Kojima T, Itoh Y, Harano Y, Fujii K, et al. (2007) The severity of ultrasonographic findings in nonalcoholic fatty liver disease reflects the metabolic syndrome and visceral fat accumulation. Am J Gastroenterol 102 2708-2715.

20. Liang RJ, Wang HH, Lee WJ, Liew PL, Lin JT, et al. (2007) Diagnostic value of ultrasonographic examination for nonalcoholic steatohepatitis in morbidly obese patients undergoing laparoscopic bariatric surgery. Obese Surg 17: 45-

21. Mishra P, Younossi ZM (2007) Abdominal ultrasound for diagnosis of nonalcoholic fatty liver disease (NAFLD). Am J Gastroenterol 102: 2716-2717.

22. Sanyal AJ, Campbell-Sargent C, Mirshahi F, Rizzo WB, Contos MJ, et al. (2001) Nonalcoholic steatohepatitis: association of insulin resistance and mitochondrial abnormalities. Gastroenterology 120: 1183-1192.

23. Seki S, Kitada T, Yamada T, Sakaguchi H, Nakatani K et al.(2002) In situ detection of lipid peroxidation and oxidative DNA damage in non-alcoholic fatty liver disease. J Hepatol 37: 56-62.

24. Chalasani N, Deeg MA, Crabb DW (2004) Systemic levels of lipid peroxidation and its metabolic and dietary correlates in patients with nonalcoholic steatohepatitis. Am J Gastroenterol 99: 1497-1502.

25. Videla LA, Rodrigo R, Orellana M, Fernandez V, Tapia G, et al. (2004) Oxidative stress-related parameters in the liver of non-alcoholic fatty liver disease patients. Clin Sci (Lond) 106: 261-268.
26. MacDonald GA, Bridle KR, Ward PJ, Walker NI, Houglum K, et al. (2001) Lipid peroxidation in hepatic steatosis in humans is associated with hepatic fibrosis and occurs predominately in acinar zone 3. J Gastroenterol Hepatol 16: 599 606.

27. Pessayre D, Berson A, Fromenty B, Mansouri A (2001) Mitochondria in steatohepatitis. Semin Liver Dis 21: 57-69.

28. Menendez JA, Lupu R (2007) Fatty acid synthase and the lipogenic phenotype in cancer pathogenesis. Nat Rev Cancer 7: 763-777

29. Grundy SM, Brewer HB, Cleeman JI, Smith SC Jr, Lenfant C. (2004) Definition of metabolic syndrome: Report of National Heart, Lung and Blood Institute/ American Heart Association conference on scientific issues related to definition. Circulation 109: 433-438

30. Marchesini G, Brizi M, Morselli-Labate AM, Bianchi G, Bugianesi E, et al. (1999) Association of nonalcoholic fatty liver disease with insulin resistance. Am J Med 107: 450-455

31. Matsuzaka T, Shimano H. (2011) Molecular mechanisms involved in hepatic steatosis and insulin resistance. J Diabetes Invest 2: 170-175

32. Farrell GC, Larter CZ (2006) Nonalcoholic fatty liver disease: from steatosis to cirrhosis. Hepatology 43: S99-99S112.

33. Clark JM (2006) The epidemiology of nonalcoholic fatty liver disease in adults $\mathrm{J}$ Clin Gastroenterol 40: S5-10. 